\title{
Impact of Mitral Regurgitation on Clinical Outcomes After Transcatheter Aortic Valve Implantation
}

\author{
Crochan J O’Sullivan, David Tüller, Rainer Zbinden and Franz R Eberli
}

Department of Cardiology, Triemli Hospital, Zurich, Switzerland

\begin{abstract}
Severe aortic stenosis (AS) and mitral regurgitation (MR) are the two most common valvular lesions referred for surgical intervention in Europe and frequently co-exist. In patients with both severe AS and significant MR referred for surgical aortic valve replacement (SAVR), a concomitant mitral valve intervention is typically performed if the MR is severe, despite the higher associated perioperative risk. The management of moderate MR among SAVR patients is controversial and depends on a number of factors including MR aetiology (i.e., organic versus functional MR), feasibility of repair and patient risk profile. Moderate or severe MR is present in up to one-third of patients undergoing transcatheter aortic valve implantation (TAVI), is mainly of functional aetiology and is typically left untreated. Although data are conflicting, a growing body of evidence suggests that significant MR exerts an adverse effect on both short- and long-term clinical outcomes after TAVI. Moderate or severe MR improves in just over half of patients following TAVI and recent data suggest MR is more likely to improve among patients receiving a balloon-expandable as compared with a self-expandable transcatheter heart valve.
\end{abstract}

\section{Keywords}

Aortic stenosis, mitral regurgitation, aortic valve replacement, transcatheter aortic valve implantation

Disclosure: FRE has received institutional grants from Abbott Vascular, Biotronik and Terumo. The other authors have no conflicts of interest to declare. Received: 29 February 2016 Accepted: 5 April 2016 Citation: Interventional Cardiology Review, 2016;11(1):54-8 DOI: 10.15420/icr.2016:11:1 Correspondence: Crochan O'Sullivan, MD, PhD, Department of Cardiology, Stadtspital Triemli, Birmendorferstrasse 497, 8063 Zurich, Switzerland. E: crochan.osullivan@triemli.zuerich.ch

Aortic stenosis (AS) is the most frequent form of valvular heart disease referred for surgery in Europe and is the second most prevalent form of valvular heart disease in the US. ${ }^{1,2}$ Conversely, moderate or severe mitral regurgitation (MR) is the most prevalent valvular disease in the US and the second most common form of valvular heart disease requiring surgery in Europe. ${ }^{1,2} \mathrm{AS}$ and MR frequently coexist and the prevalence of both valvular lesions increases with age. ${ }^{2,3}$ While AS imposes a pressure overload on the left ventricle (LV) leading to LV concentric hypertrophy, ${ }_{1}^{4}$ MR exerts a volume overload leading to progressive LV and LA dilatation. ${ }^{5}$ Indeed, severe AS may create or even worsen MR by increasing the LV to left atrial pressure gradient thereby augmenting the regurgitant volume for any given effective regurgitant orifice. ${ }^{6}$

Both valvular lesions can lead to pulmonary hypertension and right ventricular dysfunction in isolation (severe MR more so than severe AS) but the combination of both compound the problem, resulting in hypertrophic remodeling of the pulmonary arterioles and potentially irreversibly elevated pulmonary artery pressures.? AS is mainly caused by a degenerative inflammatory process, which is typically initiated by mechanical stress of the valve leaflets. ${ }^{4}$ Conversely, MR aetiology is more heterogeneous and may be categorised as either organic (intrinsic valve lesions) or functional (structurally normal mitral valve but deformation caused by ventricular remodelling). ${ }^{5}$ Surgical aortic valve replacement (SAVR) has traditionally been considered the gold standard treatment of severe symptomatic AS but up to one-third of patients with symptomatic severe AS are denied treatment owing to advanced age and co-morbidities. ${ }^{1,8}$ Several randomised clinical trials have demonstrated TAVI to be a non-inferior or even a superior alternative to SAVR in high-risk patients with severe AS.9-11 Moderate or severe $M R$ is present in up to one-third of patients undergoing TAVI and the prevalence is even higher among certain AS subgroups such as patients with low ejection fraction, low-gradient (LEF-LG) severe AS (20-55 \%).,.12 In contrast to patients undergoing SAVR, significant MR is generally left untreated among patients assigned to TAVI (see Figure 1). ${ }^{3}$ The aim of this review is to summarise the data to date regarding the effect of significant (i.e. moderate or severe) MR on clinical outcomes after TAVI.

\section{Mitral Regurgitation Aetiology}

Organic MR is the most common aetiology of MR (60-70\%) and is frequently due to primary myxomatous disease, primary flail leaflets or calcification of the mitral valve apparatus. ${ }^{5}$ Mitral valve prolapse is an abnormal systolic valve movement into the left atrium ( $\geq 2 \mathrm{~mm}$ beyond the annular level). ${ }^{5}$ Prolapse can either be moderate (leaflet tips remain in the LV i.e. billowing mitral valve) or severe (eversion of the leaflet tip into left atrium, i.e. flail leaflet). ${ }^{5}$ The main phenotypes of mitral prolapse are diffuse myxomatous degeneration (Barlows disease) or primary flail leaflets with ruptured chordae, affecting the posterior leaflet in $70 \%$ of cases. ${ }^{5}$ The presence of a flail leaflet almost always indicates advanced disease and surgery is required even in asymptomatic low-risk patients with preserved LV function and an LV end-systolic diameter $\geq 40 \mathrm{~mm}$. ${ }^{1,13}$ Rarer causes of organic 
MR include endocarditis as well as congenital (cleft leaflet), rheumatic (acute and chronic rheumatic fever), iatrogenic (radiation/drugs) and inflammatory (lupus/anticardiolipin, eosinophilic endocardial disease, endomyocardial fibrosis) conditions. ${ }^{5}$ While organic MR is more prevalent in the general population, functional MR is more prevalent among elderly patients referred for TAVI. ${ }^{3}$ This may be related to the high prevalence of concomitant coronary artery disease (40-70\%) among severe AS patients undergoing TAVI. ${ }^{14}$ Functional MR is usually related to ischaemia and is caused by apical and inferiorpapillary-muscle displacement due to ischaemic left-ventricular remodeling. ${ }^{5}$ Papillary-muscle displacement exerts traction on leaflets because chordae are non-extensible, which results in tethered and apically displaced leaflets (tenting). Together with annular flattening, enlargement and decreased contraction, mitral valve tenting results in coaptation loss that results in functional MR. ${ }^{5}$

It should be noted that ischaemic MR is not synonymous with functional MR since ischaemic papillary muscle rupture is classified as organic MR. Non-ischaemic causes of functional MR include cardiomyopathy, myocarditis and other non-ischaemic causes of left ventricular dysfunction. ${ }^{5}$ Carpentier proposed to classify MR into three types according to leaflet movement: type I (normal movement), type II (excessive movement) and type III (restrictive movement: IIIa - diastolic restriction; IIIb systolic restriction). ${ }^{5}$ The assessment of MR severity using echocardiography comprises qualitative (e.g. valve morphology, colour flow regurgitant jet, continuous wave signal of regurgitant jet), semiquantitative (e.g. vena contracta width, systolic pulmonary vein flow reversal) and quantitative (e.g. effective regurgitant orifice area [EROA] and regurgitant volume [R Vol]) methods. ${ }^{15}$ EROA and $\mathrm{R}$ vol can be calculated by either the flow convergence or the Doppler volumetric method..$^{15}$ Severe primary MR is defined quantitatively as an EROA $\geq 40 \mathrm{~mm}^{2}$ and a $\mathrm{R}$ vol $\geq 60 \mathrm{ml}^{1.115}$ In secondary MR, lower thresholds of severity using quantitative methods, have been proposed (EROA $\geq 20 \mathrm{~mm}^{2}$ and $\mathrm{R}$ vol $\geq 30 \mathrm{ml}$ ) because of their prognostic value. ${ }^{1,15}$

Other criteria for severe MR are shown in Table 1. The assessment of AS severity is more complicated in the presence of significant MR owing to the fact that forward stroke volume is reduced due to the $\mathrm{R}$ vol " lost » in the left atrium. ${ }^{\circ}$ Mean transaortic gradient is directly proportional to the square of transvalvular flow meaning that even small reductions in stroke volume can result in significant reductions in the pressure gradient. ${ }^{16}$ This in turn can lead to guideline discordant mean gradient and aortic valve area values making the grading of AS severity challenging among patients with significant MR.

\section{SAVR and Significant MR}

Double valve interventions are associated with a higher perioperative mortality rate compared with isolated SAVR. ${ }^{17,18}$ In the Society for Thoracic Surgeons (STS) registry, the perioperative mortality after double (mitral-aortic) valve intervention was almost three-fold higher (9.4\%) compared with isolated AVR (3.2\%). ${ }^{17,18}$ The decision to intervene on the mitral valve in the setting of severe AS depends on the severity and the aetiology of MR. In symptomatic patients with severe AS, valve replacement (conventional or percutaneous) is the treatment of choice, while in patients with severe MR, valve repair is generally favoured over replacement if feasible especially among patients with organic disease. ${ }^{4.5}$ This is mainly due to the fact that mitral valve repair is associated with lower perioperative mortality, improved survival and better preservation of post-operative LV function. ${ }^{1,19}$
Figure 1: Severe Aortic Stenosis and Severe Organic Mitral Regurgitation

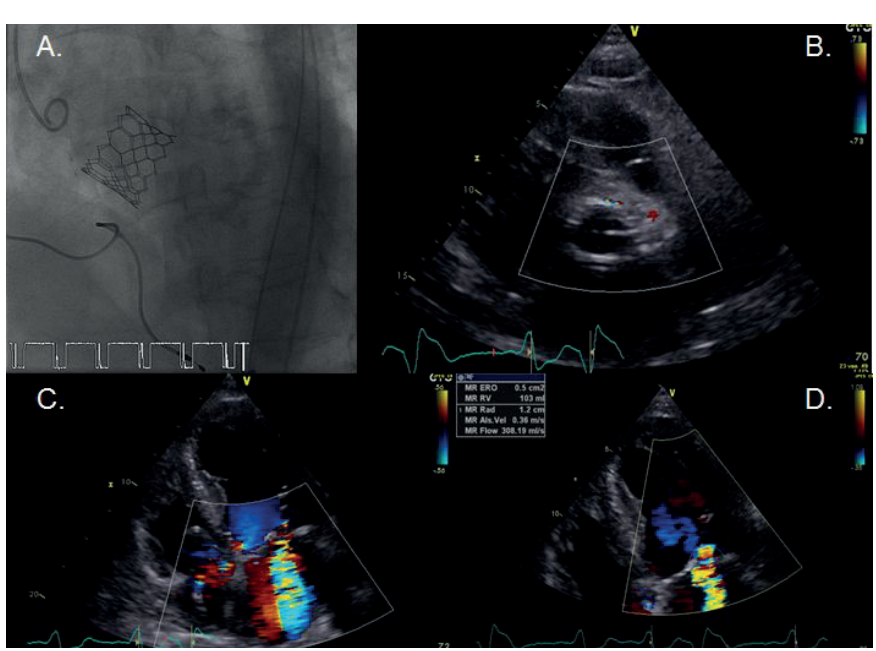

Example of a 78-year-old male patient with symptomatic severe low ejection fraction, lowgradient (LEF-LG) aortic stenosis (AS) and concomitant severe organic mitral regurgitation (MR) who underwent transcatheter aortic valve implantation (TAVI) with a $29 \mathrm{~mm}$ Edwards SAPIEN 3 bioprosthesis A. There was a minimal paravalvular aortic regurgitation following TAVI on transthoracic echocardiography; B. Colour flow mapping demonstrated severe MR persisting after TAVI; C. The severity was confirmed using quantitatively (effective regurgitant orifice area $[E R O A]=50 \mathrm{~mm}^{2}$ and regurgitant volume $=103 \mathrm{~mL}$ ); $D$. A recent study reported that patients with $L E F-L G$ ) severe AS and moderate to severe MR have a three-fold higher mortality rate as compared with similar patients with mild or less MR after TAVI. ${ }^{12}$ Organic MR (as shown in the present example) is less likely to improve after TAVI as compared with functional $M R^{12}$

Table 1: Echocardiographic Criteria for the Definition of Severe Mitral Regurgitation Using an Integrative Approach

\begin{tabular}{|c|c|c|}
\hline Characteristics & \multicolumn{2}{|c|}{ Mitral Regurgitation } \\
\hline \multicolumn{3}{|l|}{ Qualitative } \\
\hline Valve morphology & \multicolumn{2}{|c|}{$\begin{array}{l}\text { Flail leaflet/ruptured papillary muscle/large } \\
\text { coaptation defect }\end{array}$} \\
\hline $\begin{array}{l}\text { Colour flow } \\
\text { regurgitant jet }\end{array}$ & \multicolumn{2}{|c|}{$\begin{array}{l}\text { Very large central jet or eccentric jet adhering, swirling, } \\
\text { and reaching the posterior wall of the left atrium }\end{array}$} \\
\hline $\begin{array}{l}\text { CW signal of } \\
\text { regurgitant jet }\end{array}$ & \multicolumn{2}{|c|}{ Dense/triangular } \\
\hline Other & \multicolumn{2}{|c|}{ Large flow convergence zone } \\
\hline \multicolumn{3}{|l|}{ Semiquantitative } \\
\hline Vena contracta width (mm) & \multicolumn{2}{|c|}{$\geq 7$ (>8 for biplane) } \\
\hline Upstream vein flow & \multicolumn{2}{|c|}{ Systolic pulmonary vein flow reversal } \\
\hline Inflow (m/s) & \multicolumn{2}{|c|}{ E-wave dominant $\geq 1.5$} \\
\hline Other & \multicolumn{2}{|c|}{ TVI mitral/TVI aortic $>1.4$} \\
\hline Quantitative & Organic & Functional \\
\hline EROA $\left(\mathrm{mm}^{2}\right)$ & $\geq 40$ & $\geq 20$ \\
\hline R Vol (ml/beat) & $\geq 60$ & $\geq 30$ \\
\hline $\begin{array}{l}\text { enlargement of } \\
\text { cardiac chambers/ } \\
\text { vessels }\end{array}$ & \multicolumn{2}{|l|}{ LV, LA } \\
\hline
\end{tabular}

$C W=$ continuous wave Doppler; $E R O A=$ effective regurgitant orifice area; $L A=$ left atrium; $L V=$ left ventricle; $R \mathrm{VOl}=$ regurgitant volume; $T \mathrm{VI}=$ time velocity intergral.

For example, in the STS registry, perioperative mortality was $5.7 \%$ for mitral valve replacement versus just $1.6 \%$ for mitral valve repair. ${ }^{17}$ Conversely, among patients with severe functional MR of ischaemic aetiology, there appears to be no clear benefit of mitral valve repair over replacement, despite indications from earlier retrospective studies suggesting there was. ${ }^{20,21}$ A recent multicentre randomised clinical trial revealed no significant differences in left ventricular reverse remodeling or survival at 2 years among patients randomised to repair versus replacement. ${ }^{21} \mathrm{MR}$ recurred more frequently in the 
repair group, leading to more heart-failure-related adverse events and cardiovascular admissions. ${ }^{21}$ Whether these results can be extrapolated to patients with severe functional MR undergoing SAVR is uncertain. Among patients undergoing SAVR there is a general consensus that a double-valve operation is indicated in the presence of severe MR. ${ }^{5}$ The management of moderate MR at the time of SAVR is controversial. ${ }^{22-24}$

A recent meta-analysis suggested that moderate MR left untreated during SAVR may be associated with worse early and late clinical outcomes, suggesting that double-valve intervention may be indicated in such instances.22 Barreiro et al. reported a higher mortality rate among patients with moderate MR of mainly organic aetiology (63\%) left untreated at the time of SAVR. ${ }^{23}$ Among patients with moderate functional MR undergoing SAVR, Ruel et al. observed that untreated moderate functional MR had no independent adverse effect on survival at mean follow-up of $5.4 \pm 3.4$ years. ${ }^{24} \mathrm{AS}$ patients with moderate functional MR and one additional risk factor (left atrial diameter $>5 \mathrm{~cm}$, mean/peak gradient $<40 / 60 \mathrm{mmHg}$ or atrial fibrillation [AF]) were at increased risk for the composite outcome of heart failure symptoms, cardiac death or subsequent mitral repair or replacement (hazard ratio $[H R] 2.7 ; p=0.004$ ). ${ }^{24}$

In the Placement of Aortic Transcatheter Valve (PARTNER) trial, 59 of the 299 patients who underwent isolated SAVR had moderate (90.5\%) or severe (9.5\%) MR. ${ }^{25}$ As compared to patients with mild or less MR, overall mortality rate at 2 years was significantly higher among patients with moderate or severe MR (49.1\% versus $27.9 \%$; $\mathrm{p}<0.01)$. In addition, moderate or severe MR was an independent predictor of 2-year mortality in multivariate analysis (HR 1.77; $95 \%$ confidence interval [CI] [1.17-2.68]). ${ }^{25}$ However, in a recent multicentre clinical trial randomising patients undergoing CABG with moderate functional MR of ischaemic aetiology to either $\mathrm{CABG}$ alone or $\mathrm{CABG}$ plus mitral-valve repair, the addition of mitral valve repair did not improve LV reverse remodeling as compared with CABG alone and led to more adverse events. ${ }^{26}$ The prevalence of moderate or severe MR was reduced at 1 year in the repair group and whether or not this may improve outcomes over medium- to long-term follow-up remains to be seen. ${ }^{26}$

\section{Prevalence of MR in Patients Undergoing TAVI}

The prevalence of moderate to severe MR among patients undergoing TAVI ranges between 2-33\% of all patients with severe AS undergoing TAVI, but among certain subgroups, such as patients with LEF-LG severe AS, the prevalence is considerably higher (20-55\%). ${ }^{3.12}$ Only a few studies have provided data on MR aetiology in patients undergoing TAVI. ${ }^{27-34}$ While organic MR is usually more frequent than functional MR in the general population, the latter is more common than the former among high-risk patients selected for TAVI. ${ }^{3,5}$ No study to date has reported the prevalence of mixed MR aetiologies (i.e. organic + functional), but it is likely to be significant.

\section{Effect of MR on Short Term Outcomes After TAV}

There are studies which suggest an increase in early mortality after TAVI among patients with significant MR at baseline ${ }^{27,33,35-37}$ and other studies that have not observed this association. ${ }^{12,25,38,39}$ The reasons for the discrepancies are due to the different definitions used to define significant MR, with some studies evaluating severe MR only, ${ }^{35,37}$ whereas others report the effect of moderate/severe MR on outcomes after TAVI. $25,33,3,3,38,39$ Many retrospective studies may be underpowered to detect differences at 30-days owing to relatively low event rates.
Recently three meta-analysis studies have been published assessing the effect of moderate to severe MR on clinical outcomes after TAVI. ${ }^{40-42}$ First, Nombela-Franco performed a large meta-analysis of eight studies enrolling 8,015 patients (self-expandable valve $43 \%$, balloonexpandable valve $64 \%, 1 \%$ other) assessing the effect of moderate to severe MR on clinical outcomes after TAVI. ${ }^{40}$ The authors found that overall 30-day mortality rates were significantly increased in patients with moderate-to-severe MR (odds Ratio [OR] 1.49, 95 \% Cl [1.16-1.92]) although significant heterogeneity was observed across studies $(p<0.05) .{ }^{40}$ While the impact of MR on mortality was not different between self-expandable and balloon-expandable valves in metaregression analysis $(p=0.36)$ significant MR was more likely to improve among patients receiving a balloon expandable valve as compared with a self-expandable valve. ${ }^{40}$ Several factors were postulated to explain this observation including the possibility that the longer frame of the corevalve ${ }^{\mathrm{TM}}$ system could mechanically interfere with the anterior mitral valve leaflet. ${ }^{30}$ However, this putative mechanism was not confirmed in a large Corevalve registry. ${ }^{27}$ The higher prevalence of post-procedural paravalvular aortic regurgitation may maintain volume overload and contribute to less MR improvement in such patients. .33.44 $^{34}$ Furthermore, Corevalve implantation is associated with a higher rate of both left bundle branch block and permanent pacemaker implantation, which may lead to LV dysynchrony and a negative effect on LV remodeling and consequently less MR improvement. ${ }^{45}$

Second, Chakravarty et al. performed a meta-analysis of eight studies (three of which were conference abstracts only) enrolling 8,927 patients assessing the impact of moderate to severe MR on outcomes after TAVI. ${ }^{41}$ The authors observed that mild or less MR was present in $77.8 \%$ and moderate to severe MR in $22.2 \%$ of patients. ${ }^{41}$ They observed that the presence of moderate to severe MR at baseline was associated with an increased 30-day mortality rate (relative risk [RR] 1.35; $95 \%$

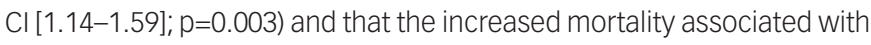
moderate-severed MR was not influenced by MR aetiology $(p=0.56){ }^{41}$

Finally, Sannino et al. performed a meta-analysis of 13 studies enrolling 4,839 patients undergoing TAVI and observed that all-cause mortality was increased at 30-days after TAVI (effect size -0.18, $95 \%$ $\mathrm{Cl}[-0.31--0.04]){ }^{42}$

\section{Effect of MR on Mid- to Long-term Outcomes After TAVI}

Data from several national registries including the German, Italian, French $^{2}$ and Spanish TAVI registries observed a significant association between moderate to severe MR and 1 year mortality after TAVI. ${ }^{27,37,46,47}$ In the French ${ }^{2}$ and Spanish TAVR registries, there remained only a trend toward higher mortality after adjustment for confounding variables in the multivariate analysis. ${ }^{37,47}$ Conversely, a post-hoc analysis of the PARTNER Cohort A trial found that moderate to severe MR at baseline did not affect 2-year mortality among TAVI patients (HR 1.14; $95 \% \mathrm{Cl}$ [0.72-1.78]; $\mathrm{p}=0.58]$ ), although it did have an impact among patients assigned to SAVR (HR 1.73; $95 \% \mathrm{Cl}[1.01-2.96] ; p=0.04) .{ }^{25}$ NombelaFranco reported in their meta-analysis that 1-year mortality rates were significantly increased among patients with moderate to severe MR (HR 1.32; $95 \% \mathrm{Cl}$ [1.12-1.55]) and that the impact of MR on mortality was not different between SEV and BEV in meta-regression analysis $(p=0.39) .{ }^{40}$

In the meta-analysis by Chakravarty et al., a strong association between moderate to severe MR and 1-year mortality after TAVI was observed (RR 1.24; $95 \%$ Cl [1.13-1.37]; $p<0.0001$ ). ${ }^{41}$ The investigators 
observed that moderate to severe residual MR after TAVI was associated with significantly increased one-year mortality (RR 1.48; $95 \% \mathrm{Cl}[1.31-1.68 ; \mathrm{p}<0.00001]) .{ }^{41}$ Finally, Sannino et al. found that moderate to severe MR was associated with both 1-year (effect size $-0.22 ; 95 \% \mathrm{Cl}[-0.36--0.08]$ ) and 2-year (effect size $-0.15 ; 95 \% \mathrm{Cl}$ [-0.27- -0.02]) mortality rates after TAVI in a meta-analysis enrolling 4,839 patients. ${ }^{42}$ The results were mainly derived from observational studies that were not specifically designed to assess the impact of MR on mortality. Additionally the PARTNER Cohort A \& B trials were the only randomised controlled trials included in these metaanalysis and concomitant severe MR was an exclusion criteria in both PARTNER trial cohorts.9.10 There was also significant heterogeneity across studies for mortality outcome. ${ }^{40-42}$ Furthermore, the aetiology of MR (functional versus organic) was not available in the majority of studies. These are the main limiting factors for the aformentioned meta-analysis studies.

\section{Effect on MR on Clinical Outcomes of Patients with Low Ejection Fraction, Low-gradient Severe AS}

Up to one in six patients undergoing TAVI present with LEF-LG severe AS and concomitant MR is present in $30-55 \%$ of these patients. ${ }^{12} \mathrm{~A}$ recent study revealed that LEF-LG patients with moderate or severe MR had a three-fold higher rate of overall mortality at one-year (11.5\% versus $38.1 \%$; adjusted HR 3.27; $95 \%$ Cl [1.31-8.15]; $p=0.011$ ), as compared with LEF-LG patients with mild or less MR. ${ }^{12}$ Patients with organic MR had higher one-year mortality rates as compared with those with functional MR (adjusted HR 3.38; $95 \%$ Cl [1.32-8.67]; $\mathrm{p}=0.011) .{ }^{12}$ However, LEF-LG patients with moderate or severe MR assigned to medical therapy had a dismal prognosis independent of MR severity suggesting that TAVI should not be withheld from symptomatic patients with LEF-LG severe AS even in the presence of moderate or severe MR. ${ }^{12}$

\section{Changes in MR Severity After TAVI}

In patients with severe AS and concomitant significant MR, several physiological changes occur following valve implantation that may contribute to reducing MR severity. The LV systolic pressure drops precipitously after TAVR/SAVR thereby reducing the LV-left atrial pressure gradient, leading to a reduction in MR in many patients. The late regression of concentric LV hypertrophy observed following TAVI due to a decrease in LV afterload can lead to favourable mitral valve haemodynamics. ${ }^{48}$ Furthermore, changes in LV geometry due to a reduction in $\mathrm{LV}$ end-diastolic volume and mitral tethering forces observed after TAVI may also lead to an improvement of functional MR (i.e. reverse remodeling). ${ }^{3}$ Nombela-Franco et al. observed that moderate to severe MR showed improvement in $51 \%$, no change in $47 \%$ and worsening in $2 \%{ }^{40}$ Reassuringly, MR appears to worsen following TAVI in only a minority of patients (2-7\%). Several studies have assessed predictors for MR improvement after TAVI.

Toggweiler et al. found that an absence of AF, absence of pulmonary hypertension (pulmonary artery systolic pressure [PASP] $<60 \mathrm{mmHg}$ ), a mean gradient $\geq 40 \mathrm{mmHg}$ and functional (as opposed to organic) MR were predictors of MR improvement following TAVI. These findings were confirmed by Bedogni et al., who also found that absence of atrial $A F$, pulmonary hypertension (PASP $\leq 55 \mathrm{mmHg}$ ) and functional MR were predictive of MR improvement after TAVI. Giordana et al. and Nombela-Franco et al. observed that valve type (BEV versus SEV) was a predictor of MR improvement. ${ }^{34,40}$ Hekimian et al. observed that an LV ejection fraction $<50 \%$, a LV end-systolic diameter $\geq 36 \mathrm{~mm}$ and an LV end-diastolic diameter $\geq 50 \mathrm{~mm}$ were predictors of MR improvement. ${ }^{32}$ The presence of prosthesis-patient mismatch leads to a residual gradient across the aortic valve and has been shown to be associated with a lesser regression of coexistant MR after SAVR. ${ }^{49-50}$ TAVI is associated with lower rates of prosthesis-patient mismatch and whether this translates into differences in MR improvement as compared with SAVR remains to be seen. ${ }^{51}$

\section{Percutaneous Treatment of MR After TAVI}

Percutaneous mitral valve repair using the Mitraclip ${ }^{\circledR}$ (Abbot Vascular) is associated with improved outcomes compared with conservative therapy in patients with symptomatic severe MR who are deemed high risk or inoperable. ${ }^{52}$ TAVI patients who remain symptomatic due to significant MR could potentially profit from a staged percutaneous procedure to treat MR. This may be a particularly attractive option for the sub-set of patients with LEF-LG severe AS and moderate to severe MR, who tend to have a particularly high mortality after TAVI. The first description of Mitraclip device being inserted as a staged procedure after TAVI with the Edwards and Corevalve devices was in 2011.53,54 The aortic valve bioprothesis did not seem to interfere with Mitraclip implantation. There is a lack of evidence on the clinical benefits associated with this procedure in the TAVI patient population. However it seems to be technically feasible and it may be a low-risk therapeutic option for patients with significant MR who remain symptomatic after TAVI.

\section{Management Strategy of Patients with Significant MR Undergoing TAVI}

The management strategy of patients with severe AS and concomitant moderate or severe MR depends on a number of factors including operative risk, MR severity, MR aetiology and likelihood of improvement. Key to decision-making is the evaluation of MR aetiology and severity by quantitative echocardiographic methods, with the use of transoesophageal echocardiography if necessary. Among low or intermediate risk patients with moderate or severe $\mathrm{MR}$, patient selection is critical to identify patients in whom MR will not improve or even progress after SAVR. In such patients with a low likelihood of MR improvement (e.g. patients with severe MR due to a flail leaflet) the increased risk of a double-valve procedure may be worthwhile. Among high risk patients in whom both SAVR and TAVI are options, identification of factors associated with improvement may lead one to choose one procedure over another. Therefore, patients with a high likelihood of MR improvement after the intervention (e.g. patients with low ejection fraction and functional MR) might be selected to undergo TAVI, whereas a combined SAVR and mitral valve repair or replacement may be a more attractive option in patients with a low chance of MR improvement after TAVI. Among inoperable patients, TAVI is the best option if feasible, especially among patients with concomitant MR of functional aetiology. Among inoperable patients who remain symptomatic due to severe MR even after TAVI, percutaneous repair of the mitral valve could be considered, although data remain scarce regarding the feasibility of this approach. ${ }^{53-56}$

\section{Conclusion}

Moderate to severe MR is commonly present among patients selected to undergo TAVI and is associated with an increased risk of both early and late mortality after TAVI. However, because of the limitations of the data hitherto available, it is not clear whether this association is related to the MR severity or whether MR is simply a marker of a worse prognosis. Patients with significant MR tend to have worse 
baseline characteristics including poorer LV function and it may not be possible to adjust for all confounders. MR severity typically improves after TAVI in about half of patients and worsens in only a small minority of patients. In the remaining patients, MR severity remains the same. Future study areas include randomised controlled clinical trials assessing the feasibility of percutaneous management of concomitant moderate to severe MR versus medical management among patients undergoing TAVI. Such studies will require centralised core laboratories to define the precise mechanism and severity of MR using quantitative methods and standardied methods of grading MR severity.
1. Vahanian A, Ottavio A, Andreotti F, et al. Guidelines on the management of valvular heart disease (version 2012). The joint task force on the management of valvular heart disease of the European Society of Cardiology (ESC) and the European Association for Cardio-Thoracic Surgery (EACTS). Eur Heart J 2012;33:2451-96. DOI: 10.1093/eurheart//ehs109: PMID: 2292241

2. Nkomo VT, Gardin MJ, Skelton TN et al. Burden of valvular heart diseases: a population-based study. Lancet 2006;368:1005-11. PMID: 16980116

3. Nombela-Franco L, Ribeiro HB, Urena M, et al. Significant mitral regurgitation left untreated at the time of aortic valve replacement. A comprehensive review of a frequent entity in the transcatheter aortic valve replacement era. I Am Col Cardiol 2014;63:2643-58. DOI: 10.1016/j.jacc.2014.02.573; PMID: 24681140

4. Carabello BA, Paulus WJ. Aortic Stenosis. Lancet 2009;373:956-66. doi: 10.1016/S0140-6736(09)60211-7; PMID: 19232707

5. Enriquez-Sarano M, Akins $\mathrm{CW}$, Vahanian A. Mitral regurgitation. Lancet 2009;373:1382-94. DOI: 10.1016/S01406736(09)60692-9; PMID: 19356795

6. Unger P, Dedobbeleer C, Van Camp G et al. Mitral regurgitation in patients with aortic stenosis undergoing valve replacement. Heart 2010;96:9-14. DOI: 10.1136/ valve replacement. Heart 2010;96:9

7. O'Sullivan CJ, Wenaweser P, Ceylan $\mathrm{O}$, et al. Effect of pulmonary hypertension hemodynamic presentation on clinical outcomes in patients with severe symptomatic aortic valve stenosis undergoing transcatheter aortic valve implantation: Insights from the new proposed pulmonary hypertension classification. Circ Cardiovasc Interv 2015:8:e002358. DOI: 10.1161/ CIRCINTERVENTIONS.114.002358; PMID: 26156149

8. Iung $B$, Baron $G$, Butchart $E G$, et al. $A$ prospective survey of patients with valvular heart disease in Europe: The euro hea survey on valvular heart disease. Eur Heart J 2003;24:1231-43. PMID: 1283181

9. Leon MB, Smith CR, Mack M, et al. Transcatheter aortic-valve implantation for aortic stenosis in patients who canno undergo surgery. N Eng/ J Med 2010;363:15

10. Smith CR, Leon MB, Mack MJ, et al. Transcatheter versus surgical aortic-valve replacement in high-risk patients. N Eng/ Med 2011;364:2187-98. DOI: 10.1056/NEJMoa1103510; PMID 21639811

11. Adams DH, Popma JJ, Reardon MJ, et al. Transcatheter aorticvalve replacement with a self-expanding prosthesis. N Eng/ I Med 2014;370:1790-8. DOI: 10.1056/NEJMoa1400590; PMID: 24678937

12. O'Sullivan CJ, Stortecky S, Bütikofer A, et al. Impact of mitral regurgitation on clinical outcomes of patients with low-ejection fraction, low-gradient severe aortic stenosis undergoing transcatheter aortic valve implantation. Circ Cardiovasc Interv 2015;8:e001895. DOl: 10.1161/ CIRCINTERVENTIONS.114.00189; PMID: 2565731

13. Ling $L H$, Enriquez-Sarano M, Seward JB, et al. Clinica outcome of mitral regurgitation due to flail leaflet. $N$ Eng/ J Med 1996;335:1417-23. PMID: 8875918

14. O'Sullivan $\mathrm{CJ}$, Stefanini $\mathrm{GG}$, Stortecky S, et al. Coronary revascularization and TAVl: before, during, after or never? Minerva Med 2014;105:475-85. PMID: 25274461

15. Lancellotti P, Moura L, Pierard LA, et al. European association of echocardiography recommendations for the assessment of valvular regurgitation. Part 2: mitral and tricuspid regurgitation (native valve disease). Eur J Echocardiogr 2010:11:307-32. DOI: 10.1093/ejechocard/jeq031: PMID: 20435783

16. O'Sullivan CJ, Praz F, Stortecky S, Windecker S, Wenaweser P. Assessment of low-flow, low-gradient, severe aortic stenosis: an invasive evaluation is required for decision making. Eurolntervention 2014; SupplU:U81-8. DOI: 10.4244/EIJV10SUA9; PMID: 25256333

17. O'Brien SM, Shahian DM, Filardo G, et al. The Society of Thoracic Surgeons 2008 cardiac surgery risk models: Part 2 - isolated valve surgery. Ann Thorac Surg 2009;88:S23-42. DO 10.1016/i.athoracsur.2009.05.056; PMID: 19559823

18. Rankin JS, He X, O'Brien SM, et al. The Society of Thoracic Surgeons risk model for operative mortality after multiple valve surgery. Ann Thorac Surg 2013;95:1484-90. DOI: valve surgery. Ann Thorac surg 2013,95.1484-90. DOt:

. ThouranivH, Suri RM, Rankin IS, et al. Doss mitral valve repar offer an advantage over replacement in patients undergoing aortic valve replacement? Ann Thorac Surg 2014 DOI: 10.1016/j.athoracsur.2011.06.083; PMID: 21983074

20. Reece TB, Tribble CG, Ellmann PI, et al. Mitral repair is
superior to replacement when associated with coronary artery disease. Ann Surg 2004;239:671-5. PMID: 15082971; PMCID: PMC1356275

21. Goldstein D, Moskowitz AJ, Glijns AC, et al. Two-year outcomes of surgical treatment of severe ischemic mitra regurgitation. N Eng/ J Med 2016;374:344-53. DOI: 10.1056/ NEJMoa1512913; PMID: 26550689

22. Harling L, Saso S, Jarral OA, et al. Aortic valve replacement for aortic stenosis in patients with concomitant mitral regurgitation: should the mitral valve be dealt with? Eu $J$ of Cardiothorac Surg 2011;40:1087-96. DOI: 10.1016/j. ejcts.2011.03.036; PMID: 21570860

23. Barreiro CJ, Patel ND, Fitton TP, et al. Aortic valve replacement and concomitant mitral valve regurgitation in the elderly: Impact on survival and functional outcome circulation 2005;112:I-443-I-447. PMID: 16159860

24. Ruel M, Kapila V, Price J, et al. Natural history and predictors of outcome in patients with concomitant functional mitral regurgitation at the time of aortic valve replacement. circulation 2006;114:I-541-I-546. PMID: 16820634

25. Barbanti M, Webb JG, Hahn RT, et al. Impact of preoperative moderate/severe mitral regurgitation on 2-year outcome after transcatheter and surgical aortic valve replacement: Insight from the Placement of Aortic Transcatheter Valve (PARTNER) trial Coct A Circulation 2013:128:2776-84.

26. Smith PK, Puskas JD, Ascheim DD, et al. Surgical treatment of moderate ischemic mitral regurgitation. N Eng/ J Med 2014;371:2178-88. DOI: 10.1161/

CIRCAATA. regurgitation and transcatheter aortic valve replacement with the CoreValve revalving system: a multicentre registry. Circulation 2013;128;2145-53. DOI: 10.1161/

28. Tzikas A, Piazza N, van Dalen BM, et al. Changes in mitral regurgitation aftger transcatheter aortic valve implantation Catheter Cardiovasc Interv 2010;75:49-9. DOI: 10.1002/ ccd.22197; PMID: 1973926

29. Durst R, Avelar E, McCarty D, et al. Outcome and improvement predictors of mitral regurgitation after transcatheter aortic valve implantation. J Heart Valve Dis 2011;20:272-81. PMID: 21714416

30. De Chiara B, Moreo A, De Marco F, et al. Influence of Corevalve revalving system implantation on mitral valve function: an echocardiographic study in selected patients. Catheter Cardiovasc Interv 2011;78:638-44. DOI: 10.1002/ ccd.23045; PMID: 21805556

31. Samim M, Stella PR, Agostoni P, et al. Transcatheter aortic implantation of the Edwards-SAPIEN bioprosthesis: insights on early benefit of TAVR on mitral regurgitation. Int I Cardiol 2011;152:124-6. DOI: 10.1016/j.jijcard.2011.07.042; PMID: 2184007

32. Hekimian G, Detaint D, Messika-Zeitoun D, et al. Mitral regurgitation in patients referred for transcatheter aortic valve implantation using the Edwards Sapien prosthesis: mechanisms and early post-procedural changes. J Am Soc Echocardiogr 2012;25:160-65. DOI: 10.1016/j.echo.2011.10.001; PMID: 22071307

33. Toggweiler S, Boone RH, Rodès-Cabau J, et al. Transcatheter aortic valve replacement: outcomes of patients with moderate or severe mitral regurgitation. I Am Coll Cardiol 2012;59:2068-74. DOI: 10.1016/J.jacc.2012.02.020; PMID: 22483326

34. Giordana F, Capriolo M, Frea S, et al. Impact of TAVR on mitral regurgitation: a prospective echocardiographic study. Echocardiography 2013:30:250-7. DOl: 10.1111/echo.12050; PMID: 23190425

35. Rodès-Cabau J, Wegg JG, Cheung A, et al. Transcatheter aortic valve implantation for the treatment of severe symptomatic aortic stenosis in patients at very high or prohibitive surgica risk: acute and late outcomes of the multicenter Canadian experience. J Am Coll Cardiol 2010;55:1080-90. DOI: 10.1016/. jacc.2009.12.014; PMID: 20096533

36. Di Mario C, Eltchaninoff $\mathrm{H}$, Moat, et al. Transcatheter Valve Treatment Sentinel Registry (TCVT) Investigators of the EUROObservational Research Programme (EORP) of the European Society of Cardiology. The 2011-12 pilot European Sentinel Registry of Transcatheter Aortic Valve Implantation: in-hospital results in 4,571 patients. Eurolntervention 2013;8:1362-71. DOI: 10.4244/EIJV8I12A209; PMID: 23256965 Sabatè M, Canovas S, Garcia E, et al. In hospital and midterm predictors of mortality after transcatheter aortic valve implantation: data from the TAVR National Registry 2010-2011. Rev ESD Cardiol 2013;66:949-58. DOI: 10.1016/1. 2010-2013. Re .003: PMID: 24774108

38. D'Onofrio A, Gasparetto V, Napodano M, et al. Impact of preoperative mitral valve regurgitation on outcomes after transcatheter aortic valve implantation. Eur I Cardiothorac Surg
2012;41:1271-6. DOI: 10.1093/ejcts/ezr236; PMID: 2221948

39. Hutter A, Bleiziffer S, Richter $\mathrm{V}$, et al. Transcatheter aortic valve implantation in patients with concomitant mitral and tricuspid regurgitation. Ann Thorac Surg 2013;95:77-84.

40. Nombela-Franco L, Eltchaninoff H, Zahn R, et al. Clinical impact and evolution of mitral regurgitation following transcatheter aortic valve replacement: a meta-analysis Heart 2015;101:1395-405. DOI: 10.1136/heartjnl-2014-307120; PMID: 26060121

41. Chakravarty $\mathrm{T}$, Van Belle $\mathrm{E}$, Jilaihawi $\mathrm{H}$, et al. Meta-analysis of the impact of mitral regurgitation on outcomes afte transcatheter aortic valve implantation. Am I Cardiol 2015;115:942-9. DOI: 10.1016/j.amjcard.2015.01.022; PMID: 25779617

42. Sannino A, Losi MA, Schiattarella GG, et al. Meta-analysis of mortality outcomes and mitral regurgitation evolution in 4,839 patients having transcatheter aortic valve implantation for severe aortic stenosis. Am I Cardiol 2014;114:875-82. DOI: 10.1016/j.amjcard.2014.06.022; PMID: 25092192

43. Abdel-Wahab M, Mehilli J, Frerker C, et al. Comparison of balloon-expandable vs self-expandable valves in patients undergoing transcatheter aortic valve replacement: the CHOICE randomized clinical trial. JAMA 2014;311:1503-14.

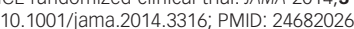

44. Jerez-Valero M, Urena M, Webb JG, et al. Clinical impact of the presence of aortic regurgitation following transcatheter aortic valve replacement: insights into the degree and acuteness of presentation. IACC Cardiovasc intv 2014:7:1022-32. DO : 10.1016/i.jcin.2014.04.012; PMID: 25234675

45. Alizadeh A, Sanati HR, Haji-Karimi M, et al. Induction and aggravation of atrioventricular valve regurgitation in the course of chronic right ventricular apical pacing. Europace 2011:13:1587-90. DOI: 10.1093/europace/eur198; PMID: 21742681

46. Zahn R, Gerckens U, Linke A, et al. Predictors of one-year mortality after transcatheter aortic valve implantation for severe symptomatic aortic stenosis. Am I Cardia 2013;112:272-9. DOI: 10.1016/j.amjcard.2013.03.024; PMID: 23578349

47. Van Belle $E$, Juthier $F$, Vincentelli $A$, et al. Does mitral regurgitation impact the outcome of TAVI procedures?
Insights from the FRANCE2 Registry. TCT-92. I Am Coll Card Insights from
2012;60:B29.

48. Gotzmann $\mathrm{M}$, Lindstaedt $\mathrm{M}$, Bojara W, Mügge A, Germing A Hemodynamic results and changes in myocardial function after transcatheter aortic valve implantation. Am Heart J 2010; 159:926-32. DOI: 10.1016/j.ahj.2010.02.030; PMID: 20435207

49. Unger P, Magne J, Vanden Eynden F, et al. Impact of prosthesis-patient mismatch on mitral regurgitation afte aortic valve replacement. Heart 2010;96:1627-32. DOI: 10.1136/hrt.2010.200428; PMID: 20937750

50. Angeloni E, Melina G, Pibarot P, et al. Impact of prosthesispatient mismatch on tthe regression of secondary mitra regurgitation after isolated aortic valve replacement with a bioprosthetic valve in patients with severe aortic stenosis. Circ Cardiovasc Img 2012;5:36-42. DOI: 10.1161/ CIRCIMAGING.111.967612; PMID: 22138006

51. Clavel MA, Webb JG, Pibarot P, et al. Comparison of the hemoynamic performance of percutaneous and surgical bioprostheses for the treatment of severe aortic stenosis. I Am Coll Cardiol 2009;53:1883-91. DOI: 10.1016/1. jacc.2009.01.060; PMID: 19442889

52. Whitlow PL, Feldman T, Pedersen WR, et al. Acute and 12-month results with catheter-based mitral valve leaflet repair: the EVEREST II (Endovascular Valve Edge-to-Edge Repair) High Risk Study. J Am Coll Cardiol 2012:59:130-9. DOI: 10.1016/j.jacc.2011.08.067: PMID: 22222076

53. Barbanti M, Ussia GP, Tamburino C. Percutaneous treatment of aortic stenosis and mitral regurgitation in the same patient: first human cases description. Catheter Cardiovasc Interv 2011;

54. Madder RD, Safian RD, Gallagher M, Senter SR, Hanzel GS. The first report of transcatheter aortic valve implantation and percutaneous mitral valve repair in the same patient. J Am Coll Cardiol Intv 2011;4:824. DOI: 10.1016/j.jcin.2011.05.009; PMID: 21777894

55. Rudolph V, Schirmer J, Franzen O, et al. Bivalular transcatheter treatment of high-surgical-risk patients with coexisting severe aortic stenosis and significant mitral regurgitation. Int Cardiol 2013;167:716-20. DOI: 10.1016/j.ijcard.2012.03.060; PMID: 22459381

56. Kische S, D'Ancona G, Paranskaya L, et al. Staged tota percutaneous treatment of aortic valve pathology and mitral regurgitation: institutional experience. Catheter Cardiovasc Inte
2013;82:E552-63. DOI: 10.1002/ccd.24809; PMID: 23359543 